Preprint typeset in JHEP style - HYPER VERSION

Imperial/TP/10/AR/01, HIP-2010-6/TH

\title{
Counting defects with the two-point correlator
}

\author{
Arttu Rajantie \\ Theoretical Physics, Blackett Laboratory, Imperial College, London SW7 2AZ, United \\ Kingdom \\ E-mail: a.rajantie@imperial.ac.uk

\section{Anders Tranberg} \\ Helsinki Institute of Physics, P.O.Box 41, FIN-00014 Helsinki, Finland and \\ Department of Physical Sciences, University of Oulu, FIN-90014, Oulu, Finland \\ E-mail: anders.tranberg@helsinki.fi
}

\begin{abstract}
We study how topological defects manifest themselves in the equal-time twopoint field correlator. We consider a scalar field with $Z_{2}$ symmetry in 1, 2 and 3 spatial dimensions, allowing for kinks, domain lines and domain walls, respectively. Using numerical lattice simulations, we find that in any number of dimensions, the correlator in momentum space is to a very good approximation the product of two factors, one describing the spatial distribution of the defects and the other describing the defect shape. When the defects are produced by the Kibble mechanism, the former has a universal form as a function of $k / n$, which we determine numerically. This signature makes it possible to determine the kink density from the field correlator without having to resort to the Gaussian approximation. This is essential when studying field dynamics with methods relying only on correlators (Schwinger-Dyson, 2PI).
\end{abstract}

KEYWORDs: Defects, out-of-equilibrium, field theory, real-time. 


\section{Contents}

1. Introduction

2. Setup, model and defects 2

3. The correlator ansatz in 1,2 and 3 dimensions 3

4. Numerical procedure 5

5. Calibration and results 5

6. Conclusion 8

\section{Introduction}

Spontaneously broken global and local symmetries play an important role in many physical systems, from high energy physics to condensed matter. In many cases, the symmetry breaking pattern is topologically non-trivial, giving rise to a possibility of topological defects, non-linear objects which are stable because of their topology. Examples of these include domain walls in ferromagnets, vortices in superfluids and superconductors, and magnetic monopoles and cosmic strings in high energy physics and cosmology.

In general, spontaneously broken symmetries are restored at sufficiently high temperatures. As the system cools down, it undergoes a phase transition into the broken phase. When this happens, the system locally picks out one of the possible vacuum states. This choice is dynamical and random, and would generally have different outcome in different patches separated by more than one correlation length. This phenomenon, known as the Kibble mechanism, results in the creation of topological defects [1, 2, 3]. It has been studied in detail in many experimental setups 4 , 5, 6, 7, 8, 9, 10, 11, 12, 13, and it may have also taken place in the early universe, producing domain walls, cosmic strings or magnetic monopoles.

The number of defects created in such a transition depends on the dynamics of the model, the nature of the symmetry and the cooling rate, which in the cosmological context is determined by the Hubble rate $H$. This dependence can be estimated by considering the critical behaviour the theory and expressed in terms of its critical exponents [2], and these estimates have been shown to be accurate in some condensed matter experiments [9, 10] and lattice field theory simulations [14, 15, 16, 17]. In these simulations, the classical equations of motion are solved numerically on a spatial lattice, and the number of defects was determined by identifying them in the final field configuration and counting them. This 
approach is fully non-perturbative and can be used also in gauge field theories [18, 19, but it cannot incorporate quantum mechanical effects and even in a classical theory, any noise such as thermal fluctuations can make the defects hard to distinguish, and the counting ill defined.

Because of these limitations, it would be advantageous to study defect formation from first principles using other techniques that would give a more detailed understanding of the process and would be valid also in quantum theory. There have been many attempts to do this using various techniques ranging from a linear approximation [20, 21, 22, 23] to Schwinger-Dyson equations, Hartree approximation [24, 14] and the 2PI formalism [25].

A common feature in these alternative approaches is that one does not have access to individual field configurations, and therefore direct counting of defects is not possible. Instead, the dynamical variables are correlation functions. This raises the question of how to determine the number of defects from the field correlator. If one assumes that the field ensemble is Gaussian and identifies zeros of the field with defects, one can derive a simple expression for their density in terms of derivatives of the two-point correlator [26, 27, 28, 25]. However, because defects are non-linear objects, their presence makes the field ensemble non-Gaussian, and therefore this assumption is not justified. Indeed, numerical simulations have shown that this approach does not work [29].

In this paper, we show how kinks, domain lines and walls in 1, 2 and 3 spatial dimensions respectively, manifest themselves in the equal time two-point correlator in momentum space at a non-linear level, without relying on the Gaussian assumption. This is an extension of previous work [29], in which we showed that the $2 \mathrm{PI}$ formalism is unable to reproduce the classical signatures of global defects, at least in a $1 / N$ expansion to next-toleading order.

We will first set up a simple model describing kinks, domain walls and domain lines (section 2), and then derive the signatures in the two-point function which we will be looking for (section (3). We then perform sample lattice simulations in both 1, 2 and 3 spatial dimensions (section 4 ), and demonstrate the signatures in practice, calibrating the net defect density the a benchmark density (section 5). We conclude in section 6 .

\section{Setup, model and defects}

We consider the action of a real scalar field $\phi$ in $D$ spatial dimensions

$$
S=\int d^{D} x d t\left(\frac{1}{2} \partial_{\mu} \phi \partial^{\mu} \phi-V(\phi)\right)
$$

with a potential $V(\phi)$, so that the theory has $Z_{2}$ symmetry $(\phi \leftrightarrow-\phi)$,

$$
V(\phi)=-\frac{1}{2} \mu^{2} \phi^{2}+\frac{\lambda}{24} \phi^{4}
$$

The parameters and fields have energy dimensions $[\phi]=(D-1) / 2,[\mu]=1,[\lambda]=3-D$. The equation of motion reads,

$$
\partial_{t}^{2} \phi(x, t)+\Gamma \partial_{t} \phi(x, t)-\partial_{x}^{2} \phi(x, t)-\mu^{2} \phi(x, t)+\frac{\lambda}{6} \phi^{3}(x, t)=0 .
$$


and we have added a small damping term $\Gamma \partial_{t} \phi([\Gamma]=1)$. This will drive the system from a high temperature initial state to a cold, near-vacuum final state (see also section (1).

The model has two degenerate vacua at $\phi_{0}= \pm v= \pm \sqrt{6 / \lambda} \mu$, and there are topological defects, kinks $(D=1)$, domain lines $(D=2)$, domain walls $(D=3)$, which interpolate between them. The classical kink solution is

$$
\phi_{\text {kink }}(x)=v \tanh \frac{x}{d}
$$

where $d=\sqrt{2} / \mu$ is the kink thickness. Domain lines and domain walls are extensions in 1 and 2 dimensions of kinks, possibly with curvature on a length scale which we will assume to be much larger than $d$.

In the following, we consider a situation in which a network of defects has been formed in a symmetry breaking phase transition by the Kibble mechanism. In this case, the defect network has one characteristic length scale, which is determined by the field correlation length at the time of the transition. It corresponds to the typical distance between defects or, equivalently, the number density $n$ of defects.

\section{The correlator ansatz in 1, 2 and 3 dimensions}

Our basic observable is the two-point correlator

$$
G(r=|x-y|)=\langle\phi(x) \phi(y)\rangle,
$$

assumed here to be homogeneous and isotropic, and therefore a function of the relative position $r$ only. The only two scales in the system are the kink thickness $d$ (or $\mu$ ), and the defect density $n^{1}$. In general, we would expect the position and momentum space correlators to be some general functions $G(r, d, n)$ and $G(k, d, n)$. In [29], we argued that in $1 \mathrm{D}$, in the limit of $d=0$, and if kinks are distributed at random with average density $n$, the correlator should have the form

$$
G(r, n)=v^{2} e^{-2 n r}, \quad G(k, n)=\frac{v^{2}}{2 n} \frac{2}{1+(k / 2 n)^{2}} .
$$

If the kinks all have the same smooth profile $\phi_{\text {kink }}(x)$, this modifies the correlator by a multiplicative factor,

$$
G(k, d, n)=\frac{v^{2}}{2 n} \frac{2}{1+(k / 2 n)^{2}} \frac{k^{2}}{4}\left|\phi_{\mathrm{kink}}(k)\right|^{2},
$$

where $\phi_{\text {kink }}(k)$ is the Fourier transform of the kink profile (2.4),

$$
\phi_{\text {kink }}(k)=\frac{2 i v}{k} \frac{\frac{1}{2} \pi k d}{\sinh \frac{1}{2} \pi k d} .
$$

\footnotetext{
${ }^{1}$ Related in a complicated way to the damping rate $\Gamma$.
} 
Therefore we should find

$$
G(k, d, n)=\frac{v^{2}}{2 n} \frac{2}{1+(k / 2 n)^{2}}\left(\frac{\frac{1}{2} \pi k d}{\sinh \left(\frac{1}{2} \pi k d\right)}\right)^{2} .
$$

Since $d$ and $v$ are known ${ }^{2}$, this expression allows for a one-parameter fit in $n$. More generally, the field correlator factorises as

$$
G(k, d, n)=\frac{v^{2}}{n} G_{\mathrm{corr}}(k / n) G_{\mathrm{kink}}(k d),
$$

where $G_{\text {corr }}(k / n)$ describes the spatial distribution of the kinks and $G_{\text {kink }}(k d)$ the kink shape. In particular, when the kinks are formed by the Kibble mechanism, the only relevant scale is their density, and therefore $G_{\text {corr }}$ is a function of the dimensionless combination $k / n$ only.

We will generalise this procedure and instead make the ansatz that the field correlator factorises in the same way in higher dimensions,

$$
G(k, d, n)=\frac{v^{2}}{n^{D}} G_{\mathrm{corr}}^{D}(k / n) G_{\mathrm{kink}}(k d) .
$$

Several comments are in order at this point. Firstly, the $d$ and $n$ dependence are separated, and apart from the trivial scaling $n^{-D}$ coming from the measure in the Fourier transform, the correlator only depends on the dimensionless quantities $k d$ and $k / n$. Also, we can obtain the kink correlation function, as

$$
G_{\text {corr }}(k / n) \rightarrow \frac{n^{D}}{v^{2}} \frac{G(k, d, n)}{G_{\text {kink }}(k d)} .
$$

The Kibble mechanism predicts that this is a universal function of the ratio $k / n$ only, and therefore if we measure this for different parameters and different cooling rates, the results should coincide. We will use numerical simulations to demonstrate that this is indeed the case, and that to a very good approximation, the universal function (in coordinate space) is of the form (for some $a_{i}, b_{i}$ )

$$
G_{\mathrm{corr}}(n r)=\left(a_{1} e^{-a_{2}(n r)^{2}}+b_{1} e^{-b_{2} n r}\right)
$$

which in D-dimensional momentum space becomes (for some other $\alpha_{i}, \beta_{i}$ )

$$
G_{\mathrm{corr}}^{D}(k / n)=\left(\alpha_{1} e^{-\alpha_{2}(k / n)^{2}}+\frac{\beta_{1}}{\left[1+\beta_{2}(k / n)^{2}\right]^{(D+1) / 2}}\right) .
$$

We use our numerical results to determine the parameters $\alpha_{i}$ and $\beta_{i}$. After this, the form of the correlator is fixed, and the only remaining free parameter is $n$ which sets the scale. The defect density $n$ can therefore be determined directly from the correlator using a simple one-parameter fit.

\footnotetext{
${ }^{2}$ Strictly speaking, these are the vacuum values, but we will see that they are sufficient. We will comment on finite temperature effects below.
} 


\section{Numerical procedure}

To carry out the numerical calculation, we discretised the equation of motion (2.3) on a lattice of spacing $a$ and using a leapfrog algorithm with time step $a \delta t$ for the time derivative. Input parameters are lattice size $n_{x}^{D}, \delta t$, and the dimensionless $a \mu, a \Gamma, a^{D-3} \lambda$.

We choose the initial conditions at time $t=0$ to mimic the quantum vacuum state corresponding to the potential

$$
V_{\text {ini }}(\phi)=\frac{1}{2} \mu^{2} \phi^{2}
$$

Because this is a free theory, the equal-time quantum two-point functions of the field $\phi$ and its canonical momentum $\pi=\partial_{t} \phi$ are simply

$$
\langle\phi(k) \phi(q)\rangle=(2 \pi) \delta(k+q) \frac{1}{2 \omega_{k}}, \quad\langle\pi(k) \pi(q)\rangle=(2 \pi) \delta(k+q) \frac{\omega_{k}}{2}, \quad\langle\phi(k) \pi(q)\rangle=0,
$$

where $\omega_{k}=\sqrt{k^{2}+\mu^{2}}$. Our initial condition is realised by a Gaussian ensemble of field configurations which has these same two-point functions [31]. We initialise all modes, and although this corresponds to a divergent energy in the continuum limit, at finite lattice spacing it is just a specific choice. In our case, it is not crucial how well these initial conditions reproduce the actual initial quantum state, since we are only interested in the classical dynamics. For our purpose, it is sufficient that it corresponds to a state with unbroken symmetry ${ }^{3}$.

The classical equation of motion (2.3) allows us to rescale the coupling $\lambda$ to unity, suggesting that only the dimensionless ratio $\lambda / \mu^{2}$ plays a role. However, the initial conditions (4.2) remove this freedom.

We solve the time evolution of the system using the classical equation of motion (2.3) for a large number of initial configurations that are drawn from the distribution specified by Eq. (4.2). Because of the inclusion of a damping term $\Gamma$, the system will lose energy and cool down to a state close to the vacuum. However, as we are going through a symmetry breaking transition, topological defects will be created. At the end of the evolution, these will be "frozen" in, possibly with very small residual thermal fluctuation superposed. The correlator at this final time is our observable, from which we will extract the number of defects.

\section{Calibration and results}

To determine the parameters $\alpha_{i}, \beta_{i}$ in (3.10) from the numerical data, we need to have an independent measurement of the defect density $n$. We do this by direct counting of zeros. We count, configuration by configuration, all lattice points $N_{\text {points }}$ where the field value is

\footnotetext{
${ }^{3}$ Other possible choices could be a Bose-Einstein distribution (finite UV energy), or a classical thermal distribution (divergent UV energy). We found that it made very little difference to the final number of defects, as long as the initial energy density was high enough. See for instance [32, 33, 34 for studies of different initial condition prescriptions in the context of symmetry breaking and thermalisation.
} 


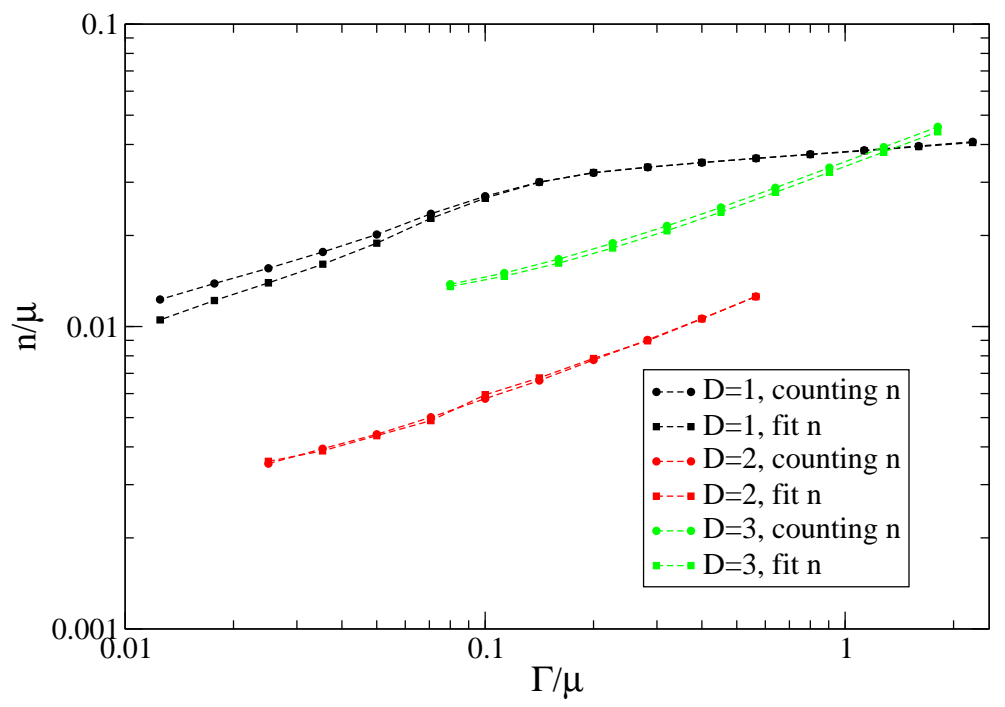

Figure 1: The "counting" defect density $n$ for different $\Gamma$ in $D=1,2,3$ dimensions (circles). Superposed, the density $n$ determined from a fit of the field correlator to our ansatz (3.10).

in some range $[-\theta, \theta]$ around $\phi=0$. We divide by the width of the kink profile in that range,

$$
\tanh [\Delta x / d]^{2}<\theta, \quad d=\sqrt{2} /(a \mu)
$$

giving for instance

$$
\begin{gathered}
\theta=0.1, \rightarrow \Delta x(\theta)= \pm 0.327 d, \quad \theta=0.05, \rightarrow \Delta x(\theta)= \pm 0.227 d, \\
\theta=0.025, \rightarrow \Delta x(\theta)= \pm 0.159 d, \quad \theta=0.0125 \rightarrow \Delta x(\theta)= \pm 0.112 d .
\end{gathered}
$$

The number of kinks/length of wall is then the number of lattice points divided by this length, and the density (in lattice units) is

$$
n(\theta)=\frac{N_{\text {points }}}{2 \Delta x(\theta) n_{x}^{D} N_{\text {configs }}} .
$$

This we consider to be the benchmark defect density, at least for late times. We checked that except at very early time, when the walls are not well separated, the kink/wall density is practically independent of the threshold $\theta$. This conclusion holds for $D=1,2,3$. The counting density as a function of damping rate is shown in Fig. 1.

For a given $\Gamma$, we calculate this "counting" density, and use it to rescale the correlator as in Eq. (3.8). If the resulting curves coincide, we will have shown that 1) the original correlator is separable in $k d$ and $k / n, 2)$ the dependence on $n$ is through $n r$ only 3) $n$ is in fact the "counting" $n$ (5.4), up to an overall calibration and 4) the rescaled curve is $G_{\text {corr }}$, which one can then attempt to approximate. Note that since the density ranges over orders of magnitude (Fig. 1), the curves certainly do not coincide without rescaling.

Fig. 2 shows the rescaled curves for various $\Gamma$ for simulations in $D=1$ spatial dimensions. We use a lattice of $2^{17}=131072$ points, $a \mu=0.1$, and $a^{2} \lambda=0.6$. We have $v^{2}=0.1$, 


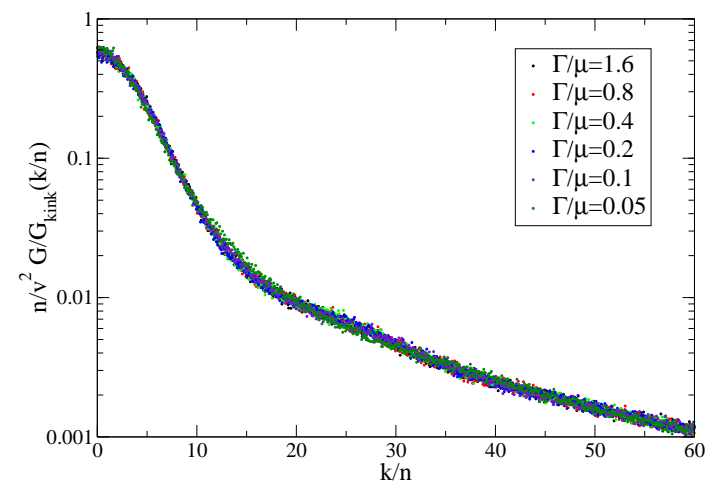

Figure 2: $\quad$ The rescaled propagators (3.8) in $D=1$ for different $\Gamma$.

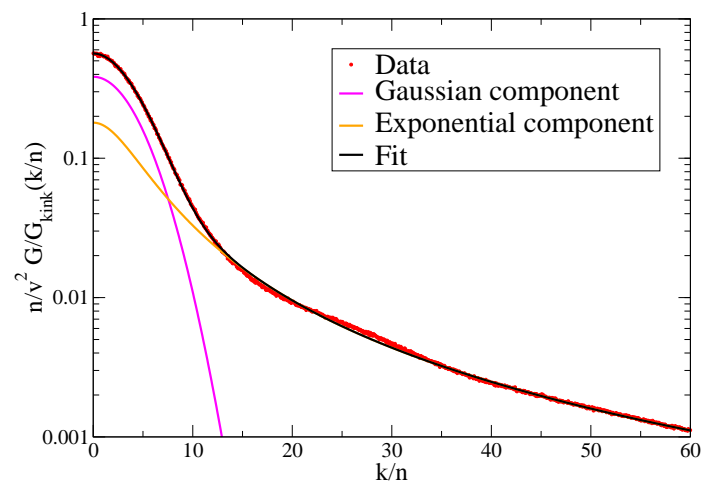

Figure 3: One rescaled propagator $(\Gamma / \mu=$ $0.1)$ with high statistics. Superposed, the fitted form (3.9), as well as the Gaussian and exponential components separately.

$d=\sqrt{2} / a \mu \simeq 14.14$. We consider a range of damping rates $\frac{\Gamma}{\mu}=0.05-3.2$. The curves agree very well indeed. In Fig. 3 we show a single rescaled curve with improved statistics (16 times as many configurations) and the overlaid fit of the anticipated form (3.9). The fit is quite convincing, although at intermediate scales, there is an oscillating feature which we choose not to account for in our description. The fit parameters $\alpha_{i}, \beta_{i}$ are given in Table 1.

\begin{tabular}{ccccc}
$D$ & $\alpha_{1}$ & $\alpha_{2}$ & $\beta_{1}$ & $\beta_{2}$ \\
\hline 1 & $0.384 \pm 0.001$ & $0.0356 \pm 0.0001$ & $0.180 \pm 0.001$ & $0.0447 \pm 0.0004$ \\
2 & $0.725 \pm 0.008$ & $0.1039 \pm 0.0006$ & $0.274 \pm 0.010$ & $0.097 \pm 0.003$ \\
3 & $1.73 \pm 0.01$ & $0.1654 \pm 0.0004$ & $0.406 \pm 0.013$ & $0.110 \pm 0.003$
\end{tabular}

Table 1: The fit parameters in (3.10) determined from the numerical data.

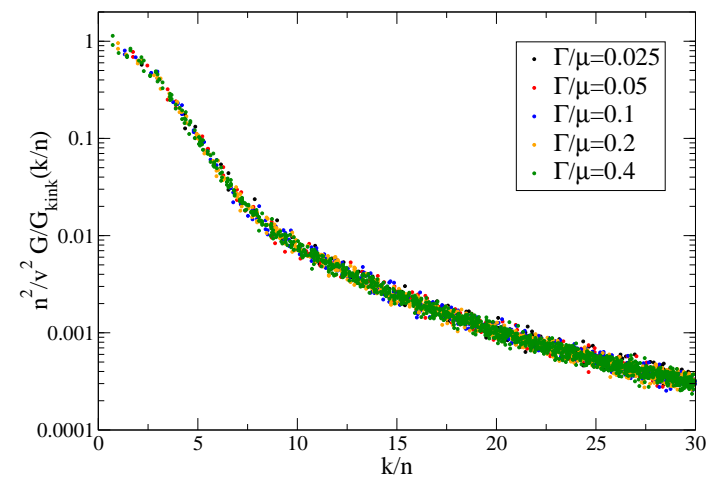

Figure 4: The rescaled propagators $(3.8)$ in $D=2$ for different $\Gamma$.

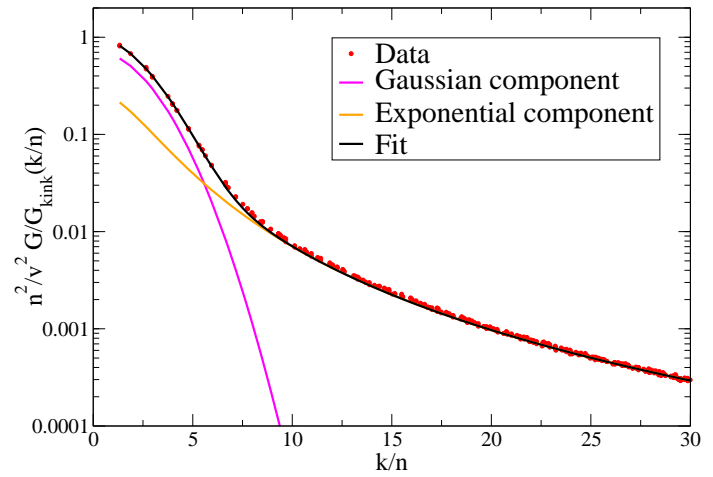

Figure 5: One rescaled propagator $(\Gamma / \mu=$ $0.1)$ with high statistics. Superposed, the fitted form (3.9), as well as the Gaussian and exponential components separately.

Similarly, Fig. 1 shows the same method applied to simulations in $D=2$. We use a 
$2048^{2}$ lattice with $a \mu=0.4, a \lambda=0.6$. We have $a v^{2}=1.6, d=\sqrt{2} / a \mu \simeq 3.535$, and we use $\frac{\Gamma}{\mu}=0.025-0.4$. Again, the rescaled curves agree very well, and again (Fig. 5) the simple form (3.9) is an excellent fit. The fit parameters $\alpha_{i}, \beta_{i}$ are given in Table 1 .

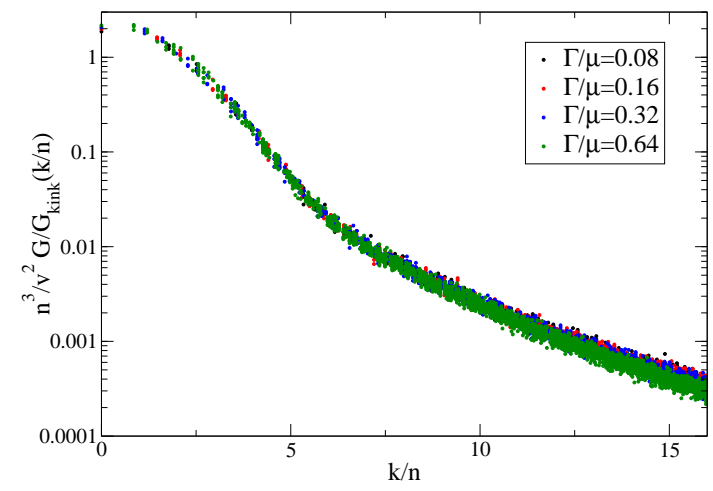

Figure 6: The rescaled propagators $(3.8)$ in $D=3$ for different $\Gamma$.

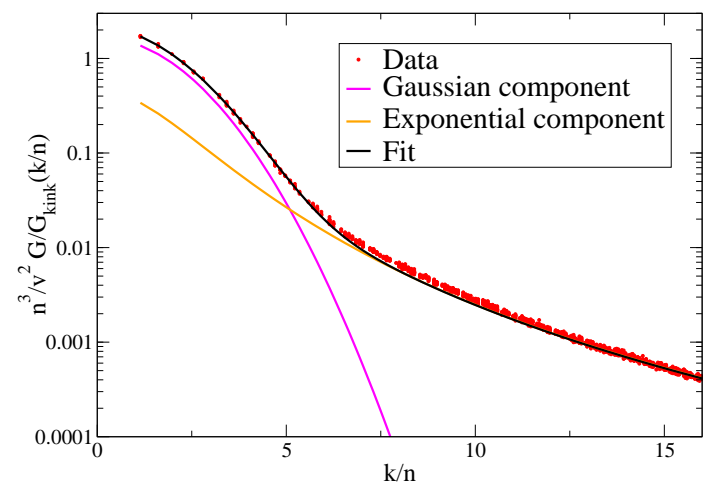

Figure 7: One rescaled propagator $(\Gamma / \mu=$ $0.1)$ with high statistics. Superposed, the fitted form (3.9), as well as the Gaussian and exponential components separately.

Finally, Figs. 6 and 7 show results in $D=3$, with a fit to the appropriate version of (3.9). We use a $256^{3}$ lattice, $a \mu=1.0, \lambda=0.6$ to give $a^{2} v^{2}=10, d=\sqrt{2} \cdot \frac{\Gamma}{\mu}=0.08-0.64$. The match is again very good, and we fidn the fit parameters $\alpha_{i}, \beta_{i}$ given in Table 1 .

For the very large $\Gamma$ curve (magenta), the agreement in the UV is not perfect, a result of having a large defect density, $n \simeq 0.08, n d \simeq 0.11$. This means that 11 percent of all lattice points are in the core of a wall, and it is therefore not surprising that our separation of scales does not hold. The wall profile is no longer a simple tanh of $r / d$. But in the IR the agreement is still good, and for all smaller damping rates even the UV performs well.

With the parameters $\alpha_{i}, \beta_{i}$ in (3.10) known, it is possible to determine the defect density $n$ from the correlator by a simple fit to (3.10), keeping only $n$ as the free parameter. We demonstrate this by calculating $n$ as a function of $\Gamma$ in this way. Fig. 1 shows the "counting" densities $n$ and the "fit" $n$, resulting from this procedure. The agreement is excellent (within 2 percent), except for very small damping rates $\Gamma$ in $1 \mathrm{D}$, where presumably residual thermal noise introduces the discrepancy. Note that for each $D$, the calibration was performed with a separate, high-statistics, simulation at one particular value of $\Gamma$. These are not included in Fig. 1, and so although because of the successful rescalings Figs. 2, 4, 6 the match could be anticipated, it is a non-trivial result.

\section{Conclusion}

We have shown that the equal-time momentum space two-point field correlator in a defect configuration produced by the Kibble mechanism separates in an UV part encoding the kink profile (in terms of $d$ ) and an IR part encoding the distribution of kinks (in terms of the density $n$ ). We carried out numerical simulations to obtain a very good approximation to the exact form, which turned out to be the sum of a Gaussian and a position-space 
exponential. This is a somewhat surprising result, since for instance assuming randomly distributed kinks in $D=1$ yields only the exponential. Furthermore, we found that the IR part of the correlator only depends on the defect density and scales with it in a simple way. This is a highly non-trivial result and allows us to determine the defect density with a one-parameter fit to the field correlator.

This is important because direct counting of defects, which usually works well in classical field theory simulations, is not a meaningful procedure in full quantum field theory because the state of the system is not described by a classical field configuration. This can be seen concretely in many non-equilibrium quantum field theory techniques, such as the 2PI formalism, which describes the dynamics in terms of the correlation functions. Our results can be employed in such calculations to determine the produced number of defects, or to check the validity of these techniques in non-linear situation. Indeed, we used this approach in an earlier work [29] to show that at next-to-leading order in the $1 / \mathrm{N}$ expansion, the $2 \mathrm{PI}$ formalism fails to describe defect formation in $1+1$ dimensions.

There is still significant room for improvement in our understanding of the defect signature in correlation functions. The present results are only valid at relatively low temperature and weak coupling. Thermal and quantum fluctuations change the form of the correlator, both by giving a direct contribution to the field correlator and by changing the kink profile [35], and more work is needed to disentangle these effects from the contribution due to the kink distribution. Other directions for future work include generalisation to other types of defects, such as vortices and monopoles.

\section{Acknowledgments}

A.T. thanks Ossi Tapio for useful discussions. A.R. was supported by STFC. The numer-

ical work was conducted on the Murska Cluster at the Finnish Center for Computational Sciences (CSC).

\section{References}

[1] T. W. B. Kibble, J. Phys. A 9 (1976) 1387.

[2] W. H. Zurek, Nature 317 (1985) 505.

[3] A. Rajantie, Int. J. Mod. Phys. A 17 (2002) 1 [arXiv:hep-ph/0108159].

[4] I. Chuang, B. Yurke, R. Durrer and N. Turok, B. Yurke, Science 251 (1991) 1336.

[5] M. J. Bowick, L. Chandar, E. A. Schiff and A. M. Srivastava, Science 263 (1994) 943 [arXiv:hep-ph/9208233].

[6] C. Baeuerle, Yu. M. Bunkov, S. N. Fisher, H. Godfrin and G. R. Pickett, Nature 382 (1996) 332.

[7] V. M. H. Ruutu et al., Nature 382 (1996) 334 [arXiv:cond-mat/9512117].

[8] S. Digal, R. Ray and A. M. Srivastava, Phys. Rev. Lett. 83 (1999) 5030 [arXiv:hep-ph/9805502].

[9] S. Ducci et al., Phys. Rev. Lett. 83 (1999) 5210. 
[10] S. Casado, Phys. Rev. E63 (2001) 057301.

[11] A. Maniv, E. Polturak and G. Koren, Phys. Rev. Lett. 91 (2003) 197001.

[12] J.R. Kirtley, C.C. Tsuei and F. Tafuri, Phys. Rev. Lett. 90 (2003) 257001.

[13] R. Monaco et al., Phys. Rev. B74 (2006) 144513.

[14] N. D. Antunes and L. M. A. Bettencourt, Phys. Rev. D 55 (1997) 925 [arXiv:hep-ph/9605277].

[15] P. Laguna and W. H. Zurek, Phys. Rev. Lett. 78 (1997) 2519 [arXiv:gr-qc/9607041].

[16] P. Laguna and W. H. Zurek, Phys. Rev. D 58 (1998) 085021 [arXiv:hep-ph/9711411].

[17] N. D. Antunes, L. M. A. Bettencourt and W. H. Zurek, Phys. Rev. Lett. 82 (1999) 2824 [arXiv:hep-ph/9811426].

[18] A. Yates and W. H. Zurek, Phys. Rev. Lett. 80 (1998) 5477 [arXiv:hep-ph/9801223].

[19] M. Hindmarsh and A. Rajantie, Phys. Rev. Lett. 85 (2000) 4660 [arXiv:cond-mat/0007361].

[20] A. J. Gill and R. J. Rivers, Phys. Rev. D 51 (1995) 6949 [arXiv:hep-th/9410159].

[21] G. Karra and R. J. Rivers, arXiv:hep-ph/9603413.

[22] G.D. Lythe, Phys. Rev. E53 (1996) R4271.

[23] M. Uhlmann, R. Schutzhold and U.R.Fischer, Phys. Rev. D81 (2010) 025017.

[24] D. Boyanovsky, D. s. Lee and A. Singh, Phys. Rev. D 48 (1993) 800 [arXiv:hep-th/9212083].

[25] G. J. Stephens, E. A. Calzetta, B. L. Hu, and S. A. Ramsey, Phys. Rev. D59 (1999) 045009, gr-qc/9808059.

[26] B. Halperin, in Physics or Defects, eds. R. Balian, M. Kleman and J. P. Poiries (North-Holland, New York, 1981).

[27] F. Liu and G. F. Mazenko, Phys. Rev. B 46 (1992) 5963.

[28] D. Ibaceta and E. Calzetta, Phys. Rev. E 60 (1999) 2999 [arXiv:hep-ph/9810301].

[29] A. Rajantie and A. Tranberg, JHEP 0611 (2006) 020, hep-ph/0607292.

[30] R. D. Blundell and A. J. Bray, cond-mat/9310075.

[31] S. Y. Khlebnikov and I. I. Tkachev, Phys. Rev. Lett. 77 (1996) 219 [arXiv:hep-ph/9603378].

[32] J. Smit, J. C. Vink and M. Salle, arXiv:hep-ph/0112057.

[33] J. Garcia-Bellido, M. Garcia Perez and A. Gonzalez-Arroyo, Phys. Rev. D 67 (2003) 103501 [arXiv:hep-ph/0208228].

[34] A. Arrizabalaga, J. Smit and A. Tranberg, JHEP 0410 (2004) 017 [arXiv:hep-ph/0409177].

[35] A. Rajantie and D. J. Weir, in progress. 